\title{
Multidetector CT Accurate for PE, but Requires Clinical Context
}

- CLINICAL QUESTION: How accurate is multidetector computed tomography for pulmonary embolism?

- BOTTOM LINE: Patients with high or intermediate probability of pulmonary embolism (PE) and an abnormal result on computed tomographic angiography (CTA) or CTA combined with venous-phase imaging (CTA-CTV) are very likely to have PE. Those with low or intermediate probability and a negative CTA or CTA-CTV result are unlikely to have PE. All other patients - that is, those with discordant findings between the clinical examination and CTA or CTA-CTV - need either further testing or close clinical followup to confirm or exclude the diagnosis. Clinical evaluation using a validated decision rule remains an important part of the evaluation. ( $\mathrm{LOE}=2 \mathrm{~b}$ )

REFERENCE: Stein PD, Fowler SE, Goodman LR, et al, for the PIOPED II Investigators. Multidetector computed tomography for acute pulmonary embolism. N Engl J Med 2006;354:2317-2327.

STUDY DESIGN: Diagnostic test evaluation

FUNDING: Government

SETTING: Emergency department

SYNOPSIS: CT technology continues to evolve, now moving from single slice CT to 4-slice or 16-slice multidetector scans. In this study, 824 patients with suspected PE underwent a standard clinical evaluation using the Wells clinical decision rule, CTA, CTA-CTV, ventilation perfusion (VQ) scanning, venous compres- sion ultrasound of the legs, and pulmonary digital subtraction angiography (DSA), if necessary. Patients were drawn from a group of 7284 patients with suspected PE, but large numbers were excluded because they couldn't complete testing within 36 hours, had abnormal renal function, declined to participate, were using anticoagulants, or were otherwise unable to complete the protocol. The mean age of participants was 51 years, $65 \%$ were white, and $62 \%$ were women. Defined by the composite reference standard (high probability VQ scan, abnormal DSA result, or abnormal venous ultrasound and nondiagnostic VQ scan), 192 (23\%) had a PE. Among those who'd had PE ruled out using this reference standard, only 2 had a likely PE during the 6-month follow-up. Also, 51 had CTA that was of insufficient quality and 87 had a CTA-CTV of poor quality, and they were excluded from the analysis. The CTA was $83 \%$ sensitive and $96 \%$ specific (positive likelihood ratio $[\mathrm{LR}+]=19.6$; negative likelihood ratio $[\mathrm{LR}-]=0.18$ ) and the CTA-CTV was $90 \%$ sensitive and $95 \%$ specific $(\mathrm{LR}+=16.5$; $\mathrm{LR}-=$ 0.11). It's important to note that the predictive value of the tests depended on the clinical assessment. The Wells rule was used to stratify patients as high, intermediate, or low risk. The positive predictive value of CTA and CTA-CTV was $96 \%$, but the negative predictive value was only $60 \%$ to $82 \%$ for those tests. However, for patients with a low clinical probability, the positive predictive value was only $57 \%$ to $58 \%$, while the negative predictive value was a robust $96 \%$ to $97 \%$. Values for positive and negative predictive value in intermedate probability patients were between $89 \%$ and $92 \%$. 